
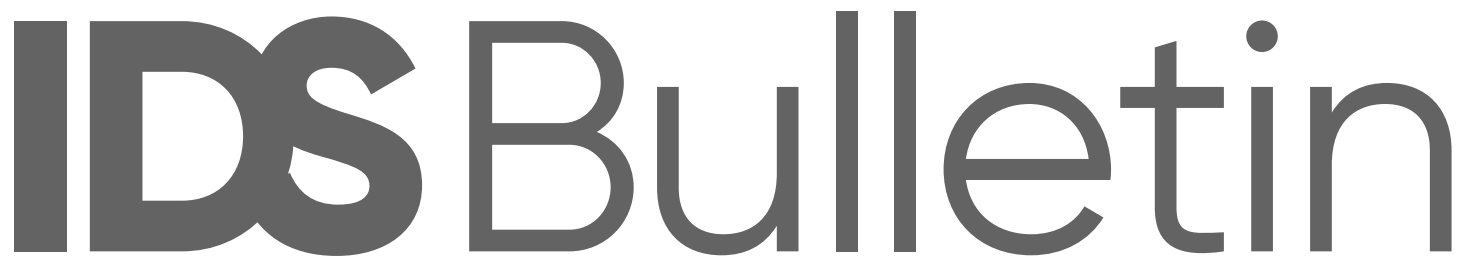

Transforming Development Knowledge

Volume 52 | Number 1 | March 2021

\title{
BUILDING A BETTER WORLD: THE CRISIS AND OPPORTUNITY OF COVID-19
}

\section{Editors Peter Taylor and Mary McCarthy}

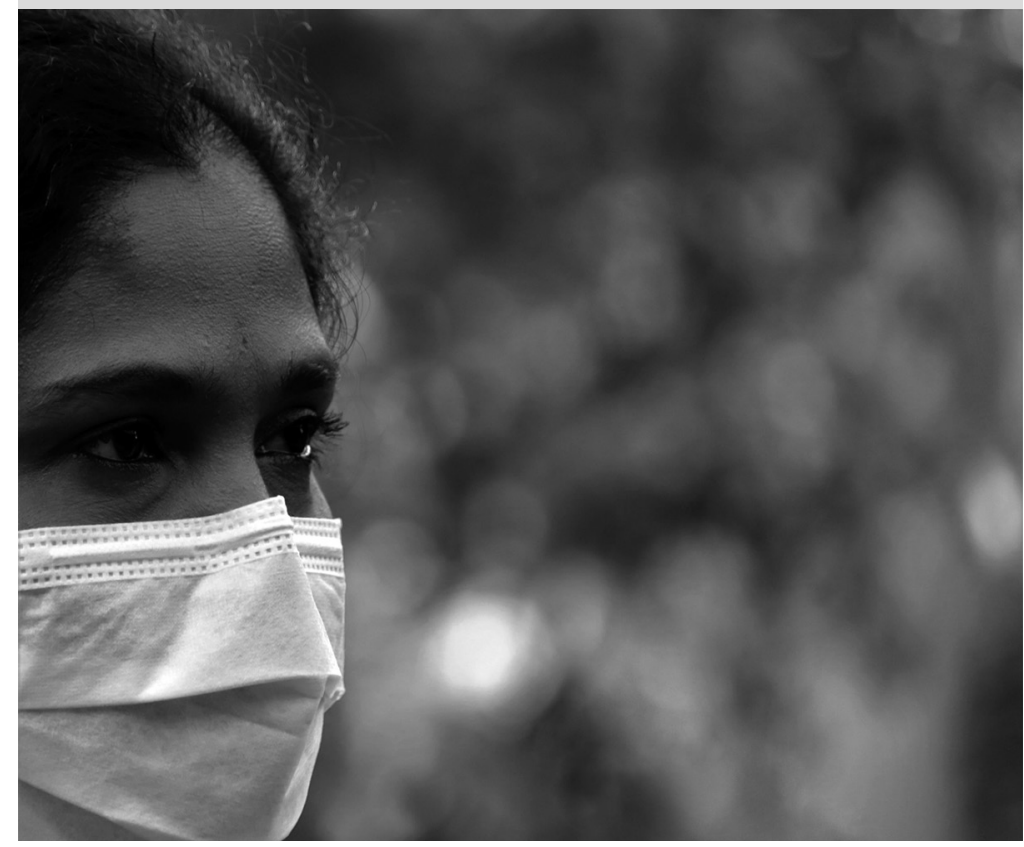


Notes on Contributors

Introduction - Building Back a Better World: The Crisis and Opportunity of Covid-19

Peter Taylor and Mary McCarthy

Local Covid-19 Syndemics and the Need for an Integrated Response Megan Schmidt-Sane, Melissa Leach, Hayley MacGregor, Jessica Meeker and Annie Wilkinson

Building Forward Better: Inclusive Livelihood Support in Nairobi's Informal Settlements Joseph Kimani, Rosie Steege, Jack Makau, Kilion Nyambuga, Jane Wairutu and Rachel Tolhurst

Social Protection, Covid-19, and Building Back Better

Jeremy Lind, Keetie Roelen and Rachel Sabates-Wheeler

Community Leaders and Decentralised Governance: Tales from the SEWA Field Paromita Sen and Aiman Haque

Food Systems After Covid-19

Ayako Ebata, Nicholas Nisbett and Stuart Gillespie

\section{Covid-19 Response and Protracted Exclusion of Informal Settlement Residents in} Freetown, Sierra Leone Abu Conteh, Mary Sirah Kamara, Samuel Saidu and Joseph Mustapha Macarthy

Building Back Better, Gender Equality, and Feminist Dilemmas

Sohela Nazneen and Susana Araujo

Beyond the Crisis: Irish Aid's Approach to Nutrition in Tanzania during the Covid-19 Pandemic

Kim Mwamelo, Peter Nyella and Adrian Fitzgerald

Religious Marginality, Covid-19, and Redress of Targeting and Inequalities Mariz Tadros, Maryam Kanwer and Jaffer Abbas Mirza

Tackling Covid-19 and Building Back Better: The Case of Ethiopia Hiwot Mebrate

Governance for Building Back Better

Shandana Khan Mohmand with contributions from Colin Anderson, Max Gallien, Tom Harrison, Anuradha Joshi, Miguel Loureiro, Giulia Mascagni, Giovanni Occhiali and Vanessa van den Boogaard 


\title{
Covid-19 Response and Protracted Exclusion of Informal Settlement Residents in Freetown, Sierra Leone"t
}

\author{
Abu Conteh, ${ }^{1}$ Mary Sirah Kamara, ${ }^{2}$ Samuel Saidu ${ }^{3}$ and \\ Joseph Mustapha Macarthy ${ }^{4}$
}

\begin{abstract}
Freetown has over 1 million residents, many of whom live in about 68 crowded informal settlements. Residents of these settlements struggle daily to access basic services such as water, sanitation, and health-care services. We found that the government's Covid-19 response measures (curfews, lockdowns, and travel restrictions) excluded informal residents from contributing to its design, and the implementation of these measures prevented these residents from accessing basic services. Like the urban planning processes in Freetown, the Covid-19 response planning was done with the limited inclusion of informal residents, and not considering how these response measures would affect their livelihood priorities. The economic conditions of already vulnerable people such as those living with disabilities, beggars, and women heads of households worsened as a result of these measures. While these challenges were dire, communities were resilient in reversing the spread of Covid-19 through tailor-made messaging and by supporting the most vulnerable with food and basic needs. In this article we argue that the inclusion of the urban poor in decision-making and urban planning processes can improve service delivery and their ability to cope with health shocks.
\end{abstract}

Keywords exclusion, informal settlements, Covid-19, vulnerability, resilience.
1 Introduction
Covid-19 is an escalation of the daily crisis that informal settlement residents in Freetown undergo to survive health, environmental, and economic vulnerabilities. Since the country's first confirmed case on 31 March 2020, and eventual spread into communities, residents of these informal settlements faced the risk of infection, the loss of livelihoods, and worsened access to health

(c) 2021 The Authors. IDS Bulletin (c) Institute of Development Studies | DOI: 10.19088/1968-2021.108 
care, water, and sanitation. This was due to the government's restriction on movement through lockdowns, curfews, and interdistrict travel restrictions which did not prioritise the participation of marginalised urban dwellers and their needs. Although Sierra Leone had two lockdowns, in April and May (lasting three days each), these restrictions impacted the livelihoods of informal settlement dwellers relying on different informal employments such as stone-breaking, small food businesses, fishing, and sand-mining. Curfews implemented over a six-month period between April and October 2020 affected small food businesses and people in the supply chain such as drivers, boat owners, and boat captains.

Similarly, inter-district travel restriction implemented from April affected food access in Freetown's informal settlements, since food supplies are mainly from the rural villages and towns. The exclusion of informal settlement residents in Freetown from participating in the planning of the Covid-19 strategy mirrors their exclusion from urban planning processes and has a long historical trajectory.

In this article, we argue that the inclusion of informal dwellers in planning is needed to utilise their knowledge of local environmental and social dynamics to better conceptualise disease pathways and effective disease prevention. People living in informal spaces in Freetown have experienced various disease outbreaks, including cholera in 2012 and the 2014-16 Ebola outbreak, and they must also contend with daily risks such as smoke from waste-burning, flood waters, rodents, mosquitoes from decomposing waste, and dust (Macarthy et al. 2018). As such, their inclusion in urban planning and epidemic response planning is imperative for designing an appropriate response to disease outbreaks.

The lack of inclusion of Freetown's urban poor in urban planning has a long history, dating back to the colonial period, when the city was divided along wealth and racial lines (Lynch, Etienne and Binns 2020). This pattern of excluding the urban poor has crossed into Sierra Leone's post-war and current marginalisation of informal settlement residents who have little say in stateled urban planning and epidemic response. Their exclusion from urban planning continues to affect the ways in which the city's services are distributed. For example, settlements around congested suburbs in eastern Freetown's precarious coastal lowlands are less serviced by the national water grid, so people look out for ingenious ways to access water.

However, this is different in the planned areas of the city where water is accessed from the national grid, and mostly at no cost (Freetown WASH Consortium 2013). Sometimes, informal service providers in these informal settlements may dig up wells or prepare a ring fence around spring-water sources from the 
hills, which they make available to residents for small fees. The money collected from the users of water sources is used to pay caretakers or buy tools for cleaning those water sources. These service access constraints take place against a backdrop of continued lack of recognition of the rights of informal settlements to exist by city authorities. The threat of eviction hinders the potential for inclusive infrastructure development for health, water, and sanitation services.

In the rest of this commentary, we reflect on how these historical trends and the resulting inadequate service provision have played out in the Covid-19 response. We report on some initial findings from remote interviews done in the first three months of the Covid-19 outbreak in Sierra Leone. Although these interviews have yet to be analysed in full, we observed that informal settlement dwellers responded in a range of ways to meet their own needs, including providing support for vulnerable groups such as those living with disabilities, women heads of households, beggars, and old people. With this article we hope to add our voice to those arguing that the exclusion of informal settlement residents disempowers them and reduces their capacity to cope with health shocks.

\section{Methods}

We conducted telephone-based interviews in Freetown between April and July 2020 in two hillside communities (Moyiba and Dwarzark) and a coastal community (Cockle Bay), to understand how residents were coping with the lockdown measures, curfews, and inter-district travel restrictions. The three communities collectively experience poor access to health and sanitation services, extreme poverty, and exposure to environmental and sanitation risks, but geographic differences such as topography affect how these health vulnerabilities are experienced. Two sets of interviews were conducted as follows:

Interviews with co-researchers: ${ }^{5}$ In total, six co-researchers resident in the communities were interviewed (two from each community) during the country's two lockdowns on questions related to the suitability of response measures, access to services, and livelihood challenges.

\section{Interviews with community residents and co-researchers:}

A total of 48 telephone-based interviews were done with 12 co-researchers and 36 community residents from different socioeconomic backgrounds. Samples selected for each of the communities included:

- four co-researchers;

three women and three men below 35 years; and

three women and three men above 35 years. 
Data from the telephone interviews were jointly analysed with co-researchers to identify community challenges and plan collective action. Data from these interviews are still being analysed and what is reported here are the initial reflections on emerging findings.

\section{Emerging findings and reflections}

Our interim findings highlight how people felt excluded from the planning of the Covid-19 response measures and how the implementation of these measures affected access to services such as health care, water, and sanitation. We further describe how response measures affected people's means of livelihood and how this increased food access vulnerabilities and other wellbeing challenges. We describe how service access vulnerabilities and loss of livelihoods affected people with social characteristics such as having a disability, being a single woman and head of household, and being an elderly person. Finally, our findings show that community resilience proved useful to the local response and helping vulnerable people cope with hardships.

\subsection{Reduced access to health care}

Poor access to health care is a harsh outcome of the Covid-19 outbreak in Freetown's informal settlements. Access to formal health care pre-Covid was a challenge for people across all ages and gender, although comparatively, access was better for beneficiaries of the free health-care initiative (pregnant and breastfeeding women, and children under five). ${ }^{6}$ However, access to these health services during Covid-19 has become more challenging, not only because people do not have money to pay for services, but due to other factors including a restriction on movement and fear of contracting Covid-19 within health-care facilities. For example, there were fears about the deliberate spread of Covid-19 by health workers and the government which increased fear about seeking medical treatment. This perception was aided by the lack of messaging from government to address misinformation in informal settlements. Restrictions on movement also affected health-seeking for people in the rugged hillside areas of the Dwarzark and Moyiba communities, which are only accessible by footpath, motor bike, or okada.? A pre-Covid-19 study shows how informal care pathways including self-medication with patent drugs and herbal remedies bought from drug peddlers and herbalists is common (Macarthy et al. 2018). Covid-19 restrictions meant that people relied further on these informal providers and practices.

Stigma against people infected with Covid-19 was also a barrier to health-seeking. For example, people were worried about the stigma of Covid-19 and would not seek care for conditions such as malaria because they did not want to be diagnosed with Covid-19 or stigmatised. Fears about being stigmatised were linked to the Ebola epidemic, when Ebola patients were stigmatised. A resident of the Cockle Bay community describes how this affected health-seeking in the community: 
Most people are afraid of going to the hospital due to the experience they got from Ebola outbreak. Majority prefer buying drugs from pharmacies or being treated at home by a health-care practitioner. (CBY-015)

Local residents who recognised the misconceptions around Covid-19 engaged in the dissemination of messages to prevent transmission and improve health-seeking. Community health workers (CHWs), chiefs, and youths volunteered to provide door-to-door messaging about health-seeking and preventive measures. Addressing these misconceptions requires collaboration among communities and epidemic response teams to improve health-seeking during epidemics. This can be done by engaging with different local actors to develop context-specific and community-led response (Leach et al. 2020).

\subsection{Food and livelihood challenges}

Many informal residents in Freetown do not have a sustained means of livelihood, and their sources of income are informal (Rigon, Walker and Koroma 2020). Informal employments are unregulated by the state and mostly lack recognition (ILO 2002). During the Covid-19 outbreak, informal workers have been affected by a loss of income due to public health measures which restricted them from working (Moussié, Alfers and Harvey 2020). For many informal workers reliant on food-based livelihoods - for example, selling street food - Covid-19-related restrictions affected them severely, and this was attributed to the government not being mindful of how response measures such as inter-district lockdowns and curfews would affect their livelihoods, as expressed by an elderly community member in Dwarzark:

Business-people are more affected because the inter-district lockdown has prevented them from going into the provinces where they buy goods for their businesses. So, they suffer, and we the customers suffer as well. (DWK 015)

In addition, low-income earners who depended on street foods for their evening meals lost secure access to food. People experiencing severe food insecurity were usually stone miners and other informal income earners who could not afford the high cost of food ingredients to prepare a daily meal. For people who could not save food within their homes, the lockdowns made the food situation worse, particularly when they had limited time to prepare. The second lockdown in May coincided with the Muslim month of fasting which caused a rapid increase in food prices. The fasting month is typically a period when there is high demand for food by people who share food with their Muslim friends, family, and neighbours. In many instances, female heads of households, particularly widows, found it difficult to cope with the food situation. A widow and a mother of five at Moyiba community who sells charcoal to make a living remarked about her ordeals to cope with food insecurity: 
As a widow and a single mother myself, it was not easy putting together food for my children to last for the three days. People are not happy, and the prices of food items are going up rapidly, so how can you manage to stock up food for a three-day lockdown? (MYB-CLPI-01)

In Dwarzark, homeless children and disabled residents were concerned that their food needs were not being met. Many of these disadvantaged people relied on non-governmental organisations (NGOs) and community groups for help, but many could not receive help during the lockdown because the NGOs themselves seemed to be struggling to support thousands of people who had been made homeless or vulnerable during the Covid-19 crisis.

For the homeless children, hunger became so dire that they broke movement regulations by begging in the neighbourhoods for food. Recognising this challenge, community elders arranged for shelter at the community centre, and asked people with surplus food to help. People with disabilities, particularly street beggars, became more vulnerable to hunger because the begging from which they made a living had been disrupted due to restrictions on gathering. People who used to help them within their communities were suffering economic hardship too.

\subsection{Water and sanitation}

Persistent lack of access to water and sanitation services are the everyday realities confronting informal residents. While they expressed a fear of being infected with Covid-19, accessing water was among their immediate concerns. Because most informal settlements are not connected to the national water grid, people access water from sources that are sometimes considered unfit for consumption. In some communities, water tanks provided by the government since the Ebola epidemic some five years ago have not been refilled. These access concerns became more severe during the lockdowns and coincided with seasonal access challenges. In Dwarzark, for example, most of the wells dried up at the peak of the dry season in April, so thousands of residents relied on a solar-powered tank which rationed water for a small fee. As a hillside community, Dwarzark residents face acute shortages of water during the dry season, when seeking water from unprotected wells and streams polluted with human waste becomes the only option (Macarthy et al. 2018). Similar access challenges apply to the Moyiba community, which has a few public taps but not enough to serve the growing population:

Water supply was a bit better before now when I was doing business, because I could purchase it without any difficulty; but now I have to either prioritise safe drinking water or food because the cost of five or six jerry cans of water is enough to prepare meal for the home. (MYB-004) 
The long-term challenges of accessing water meant that people were unable to follow basic Covid-19 guidance around handwashing and social distancing. As the water situation became acute, people searching for water ignored social distancing regulations. Hundreds clustered around the few water access points. In Moyiba, youths known as 'tap collectors' developed a strategy for enhancing fair distribution of water and to prevent people from clustering in one area. This allowed them to identify people collecting water to prevent them from receiving more than the quantity agreed for everyone. Those who could not withstand the huge crowds travelled to other communities where they felt access was relatively easier.

Access to sanitation was also a challenge since private indoor facilities are less common. The use of shared public toilets or open defecation in streams is widespread. This was a concern for maintaining social distancing because of the way people gather around these facilities. Moreover, outdoor toilets often expose women and girls to sexual exploitation and abuse, and this is also a concern because violence against women and girls has been shown to increase during conflicts and health crises such as Covid-19. Therefore, the provision of sanitation services for women and girls during a crisis must consider privacy and security to enhance utilisation (Winter, Dreibelbis and Barchi 2019). Improved data and training are required to address gendered violence around sanitation services for girls and women during health crises.

\subsection{Security concerns}

The state's response to security - including health security in informal settlements - is often informed by the notion of the residents' 'illegal tenure' status and assumptions that residents are unruly and to blame for their own problems. These views justify the use of violence against them. A police and military presence was noticeable in these communities, which caused fear. Checkpoints were mounted around the markets and main roads with frequent patrols. Some residents reported incidents of beatings and arrests of people who went in search of food and water during lockdowns. Women and girls were more likely to experience violence from security forces since they were the main collectors of household water. As a result of these vulnerabilities, there is need for a different approach, starting with a dialogue between security forces and communities to identify their priorities and build on the strengths of those communities to self-organise and self-regulate. This is particularly important during uncertain periods such as the current Covid-19 crisis (Gupte 2020).

\section{Community resilience: lessons for future responses}

Across different contexts, informal settlement dwellers experience immense pressure to meet their daily food and livelihood needs. Yet, their needs are not well considered in urban policy and planning. Within these circumstances of exclusion, and livelihood and service access constraints, the urban poor show much 
resilience especially in the face of health crises and imminent death (Rashid, Theobald and Ozano 2020). Covid-19 is an escalation of the daily crises they undergo to survive health, environmental, and economic vulnerabilities. The current Covid-19 response has shown the limited involvement of informal residents in its planning, resulting in restrictions that have caused severe hardships, particularly for already vulnerable groups such as people with disabilities, single women heading their own households, and the elderly. A lack of inclusion in urban planning and the Covid-19 response has affected their ability to meet their livelihood needs and to access health, water, and sanitation services.

In Freetown, while informal residents are excluded from urban planning processes, they have shown much intuition in responding to the current Covid-19 outbreak. With their own resources, communities have provided relief in the form of food and water for their counterparts most in need of help, and they have disseminated messages on Covid-19 to community residents (Osuteye et al. 2020). They have also organised the fair distribution of essential but scarce resources such as water in accordance with social distancing regulations. While some support has been provided by the state and city authorities, it has mostly come through state institutions and health professionals, not community organisations. This shows that there is a need for greater collaboration between the state and communities to address overlooked vulnerabilities in epidemic response, daily health risks, and service access. Without proper consultation, response measures (as in the 2014-16 West African Ebola outbreak) can have harmful effects on livelihoods, health, and wellbeing, particularly for people working and living in informal settlements (Wilkinson 2020).

\section{Conclusions}

We argue that an inclusive city planning and epidemic response are related, and that both are imperative for a socially just and resilient city. If empowered, communities can demonstrate considerable capacity to organise themselves and provide locally appropriate and tailored responses during future emergencies. Such community local actions have been amply demonstrated in Freetown's informal settlements during this Covid-19 response. We conclude that inclusive urban planning systems can empower urban informal residents and put them in control to address their own daily health risks and access to health and related services.

\section{Notes}

* This IDS Bulletin issue has been produced thanks to funding from the Government of Ireland. The opinions expressed here belong to the authors, and do not necessarily reflect those of Irish Aid or IDS. It was produced as part of the Strategic Partnership between Irish Aid and IDS on Social Protection, Food Security and Nutrition.

$\dagger$ This article was written with support from funding from the UK Global Challenges Research Fund (GCRF), and through 
collaboration with the Accountability and Responsiveness in Informal Settlements for Equity (ARISE) Hub. Many thanks to Annie Wilkinson (Institute of Development Studies) and Laura Dean (Liverpool School of Tropical Medicine) for editing the draft and providing relevant insights. Special thanks to all co-researchers who participated in data collection and analysis.

1 Abu Conteh, Urban Health Research Lead, Sierra Leone Urban Research Centre, Sierra Leone.

2 Mary Sirah Kamara, Junior Researcher, Sierra Leone Urban Research Centre, Sierra Leone.

3 Samuel Saidu, Research Assistant, College of Medicine and Allied Health Sciences, University of Sierra Leone, Sierra Leone.

4 Joseph Mustapha Macarthy, Lecturer, Institute of Geography and Development Studies, Njala University, Sierra Leone and Director, Sierra Leone Urban Research Centre.

5 Co-researchers are community residents recruited from disadvantaged population groups for the purpose of Community Based Participatory Research.

6 Pregnant women, lactating mothers, and children below five years are beneficiaries of the free health-care initiative implemented by the Government of Sierra Leone in 2010.

7 Okadas are motorbikes providing easy access to non-motorable and high traffic areas.

\section{References}

Freetown WASH Consortium (2013) Freetown WASH Consortium Final Phase I Narrative Report (accessed 10 December 2020) Gupte, J. (2020) The Emerging Lessons on Urban Vulnerability and Safety from Covid-19 in Low and Middle-Income Countries, Brighton: Institute of Development Studies (accessed 15 December 2020)

ILO (2002) Women and Men in the Informal Economy: A Statistical Picture, Geneva: International Labour Organization (accessed 10 December 2020)

Leach, M.; Meeker, J.; MacGregor, H.; Schmidt-Sane, M. and Wilkinson, A. (2020) 'Covid-19: Key Considerations for a Public Health Response', Briefing, Brighton: Institute of Development Studies (accessed 15 December 2020)

Lynch, K.; Etienne, N. and Binns, T. (2020) ' "Transforming Freetown": Dilemmas of Planning and Development in a West African City', Cities 101: 102694 (accessed 15 December 2020)

Macarthy, J.M.; Conteh, A.; Sellu, S.A. and Heinrich, L. (2018) Health Impacts of the Living Conditions of People Residing in Informal Settlements in Freetown, Freetown: Sierra Leone Urban Research Centre

Moussié, R.; Alfers, L. and Harvey, J. (eds) (2020) Informal Workers in the COVID-19 Crisis: A Global Picture of Sudden Impact and Long-Term Risk, Manchester: Women in Informal Employment: Globalizing and Organizing (accessed 10 December 2020)

Osuteye, E.; Koroma, B.; Macarthy J.M.; Kamara, S. and Conteh, A. (2020) 'The People Versus the Pandemic: Community 
Organisations in the Fight Against Covid-19 in Freetown, Sierra Leone', Urban-KNOW, 30 April (accessed 10 December 2020)

Rashid, S.F.; Theobald, S. and Ozano, K. (2020) 'Towards a Socially Just Model: Balancing Hunger and Response to the COVID-19 Pandemic in Bangladesh', BMJ Global Health 5.6: e002715 (accessed 15 December 2020)

Rigon, A.; Walker, J. and Koroma, B. (2020) 'Beyond Formal and Informal: Understanding Informalities from Freetown', Cities 105: 102848 (accessed 15 December 2020)

Wilkinson, A. (2020) 'Local Response in Health Emergencies: Key Considerations for Addressing the COVID-19 Pandemic in Informal Urban Settlements', Environment and Urbanization 32.2: 503-22 (accessed 15 December 2020)

Winter, S.; Dreibelbis, R. and Barchi, F. (2019) 'Women's Sanitation Practices in Informal Settlements: A Multi-Level Analysis of Factors Influencing Utilisation in Nairobi, Kenya', Global Public Health 14.5: 663-74 